\title{
Integrated Path Planning and Collision Avoidance for an Omni-directional Mobile Robot
}

\author{
Dong Hun Kim \\ Department of Electrical Engineering, Kyungnam University, \\ Changwon-city, Kyungnam 631-701, Korea
}

\begin{abstract}
This paper presents integrated path planning and collision avoidance for an omni-directional mobile robot. In this scheme, the autonomous mobile robot finds the shortest path by the descendent gradient of a navigation function to reach a goal. In doing so, the robot based on the proposed approach attempts to overcome some of the typical problems that may pose to the conventional robot navigation. In particular, this paper presents a set of analysis for an omni-directional mobile robot to avoid trapped situations for two representative scenarios: 1) Ushaped deep narrow obstacle and 2) narrow passage problem between two obstacles. The proposed navigation scheme eliminates the nonfeasible area for the two cases by the help of the descendent gradient of the navigation function and the characteristics of an omni-directional mobile robot. The simulation results show that the proposed navigation scheme can effectively construct a path-planning system in the capability of reaching a goal and avoiding obstacles despite possible trapped situations under uncertain world knowledge.
\end{abstract}

Key Words : autonomous robot, navigation, map building, omni-directional mobile robot, obstacle avoidance

\section{Introduction}

Navigation methods have been studied in two categories: one is a global planning based on priori complete information about the environment, and the other is a local planning based on sensory information in the uncertain environment where the location of obstacles are unknown. Global planning methods such as configuration space method, graph search method, and potential field methods guarantees to give a free path if such a path exists [1]-[4]. However, these methods are not appropriate to real-time environments where unknown obstacles may be located on a priori planned path or the location of goal may be changed. Thus, global planning seems to be more effective for off-line path planning rather than real-time strategy. Since the autonomous robots should be equipped with sensors to be able to react to unforeseen obstacles, the local approach is less computationally demanding than the global one. Therefore, local path planning has been used extensively in real-time control area [5] [6]. As a results, reactive, sensor-based approaches have been studied extensively for motion control of autonomous mobile robot in the past decade [8], [11]-[17]. However, the controllers being used in local planning have abandoned optimality in terms of the global space, in exchange for adapting to dynamic obstacles and the convergence of velocity. The controllers adopt a variety of heuristics for finding some path that approximates the trajectory suggested by the global planner. The disadvantage to these approaches is

Manuscript received Feb. 16, 2010; revised Sep. 3, 2010; accepted Sep. 7, 2010

This work was supported by Kyungnam University Foundation Grant, 2009. that the high-level structure of the path may be close to optimal, but the actual path of a autonomous robot by control actions may not be optimal.

The key advantage of local motion control over global path planning lies in their low computational complexity, which is particularly important when the environment model is updated frequently based on sensor information. The major studies of this approach are on a vector-field histogram technique [11], a dynamic window approach [13] and a potential field technique [10]. The sensor-based approaches evaluate function to determine the next action based on a current sensory input and the current state of a mobile robot system. These techniques have advantages in their efficiency of generating motion command in high frequency. However, the purely sensor-based approach ignores exploiting of global information, which allows the approach to be suboptimal. As well, most of the approaches do not completely solve the problem of mobile robots trapped in U-shaped obstacles. One of the approaches that mediates between global path planning and local constraints is the vector field histogram approach [11] and its extended version is VFH+ [21]. This approach uses a form of the potential based histograms using range measurements to determine appropriate headings. The dynamic window method [13] converts high-level plans into local controls, while accounting for dynamic obstacles. Konolige [8] suggested an approach based on dynamic programming for generating a path while obeying the kinematic constraints of a mobile robot. However, this approach contains approximation of the mobile robot as a point, and also dose not respect the dynamic constrains of the mobile robot. Ko and Simmons [16] suggested the Lane-Curvature method that extracts lanes out of a given map of the environment and modifies the evaluation function so that a robot stays on the lanes. This leads to smoother 
trajectories, especially in long corridors. The modified evaluation function according to the shape of a mobile robot was suggested for the transportation or manipulation tasks [18]. Approaches that explicitly integrate global path planning techniques with local controllers was also suggested. Burgard et al. and Thrun et al. [22],[23] suggested a path planning system to compute intermediate points lying on the optimal path. These intermediate points are transmitted to the reactive collision avoidance system, so that the mobile robot can no longer get stuck in local minima of the evaluation function.

An occupancy grid method is adopted to represent obstacles, where its representation allows adding and retrieving information about obstacles and enables easy integration of multiple sensors. The representation of obstacles by certainty levels in a grid model has been suggested by Elfes [24] in which the grid is used for off-line global path planning. Moravec and Elfes [25] also describes the use of the method for map-building. In our system the ultrasonic sensors are continuously sampled while the mobile robot is moving. If an obstacle is detected (within the predefined maximum range limit of 2 meters), the occupancy of corresponding cell are updated incrementally using an Evidential Method [26]. This method yields a more reliable obstacle representation in spite of the ultrasonic sensors' inaccuracies.

In this paper we present integrated path-planning and collision avoidance technique that takes into account the kinematics of a mobile robot and also the model of its environment. In this scheme, the autonomous mobile robot finds the shortest path by the descendent gradient of a navigation function to reach a goal. In particular, this paper presents a set of analysis for an omni-directional mobile robot to avoid trapped situations for two representative scenarios. Specifically the following cases are addressed: 1) U-shaped deep narrow obstacle and 2) narrow passage problem between two obstacles.

\section{Problem Statements: The model of an omni-directional mobile robot}

Our research has made use of omni-directional mobile robot, manufactured by the institute of physical and chemical research (RIKEN, JAPAN). The mobile robot is equipped with four omni-directional wheels which allow it to perform motion in two directions and rotate simultaneously [7] in Fig. 1(left). Three servo-systems execute the velocity control commands issued by onboard motion-controller. We have built a ring of eight ultrasonic sensors. These sensors operate at $44 \mathrm{kHz}$, and project beam with beam width of approximately 40 degrees.
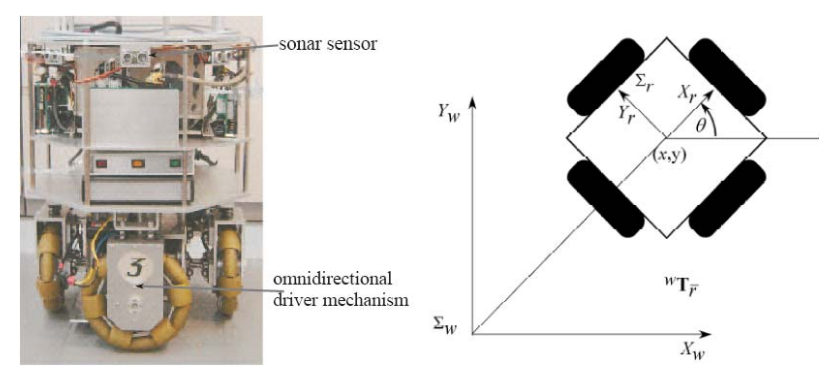

Fig. 1 The mobile robot(left) and its kinematic model(right).

The control system process the data obtained from the sensor system and the servo-systems issued the velocity control commands which are subsequently executed by the servosystems. The mobile robot is capable to perform wall-following and simple obstacle avoidance while keeping a safe distance to the object.

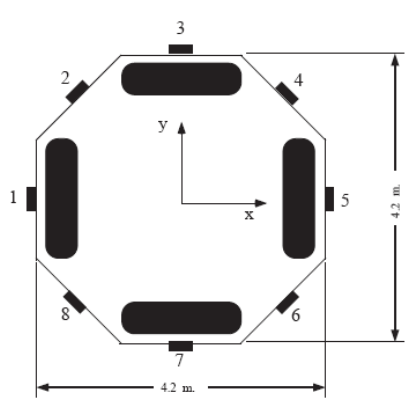

\begin{tabular}{|c|r|r|r|}
\hline Sensor & $\mathrm{x}_{\mathrm{s}}[\mathrm{m}]$ & $\mathrm{y}_{\mathrm{s}}[\mathrm{m}]$ & $\alpha_{\mathrm{s}}[\mathrm{deg}]$ \\
\hline \hline 1 & -0.210 & 0.000 & 180 \\
2 & -0.150 & 0.150 & 135 \\
3 & 0.000 & 0.210 & 90 \\
4 & 0.150 & 0.150 & 45 \\
5 & 0.210 & 0.000 & 0 \\
6 & 0.150 & -0.150 & -45 \\
7 & 0.000 & -0.210 & -90 \\
8 & -0.150 & -0.150 & -135 \\
\hline
\end{tabular}

Fig. 2 Dimensions and ultrasonic sensor locations of the mobile robot

Dimensions and ultrasonic sensor locations of the mobile robot is depicted in Fig. 2. The wheel of the mobile robot is $200 \mathrm{~mm}$ in diameter. The ultrasonic sensor ring can cover almost 360 degrees, therefore, the mobile robot can perform an omni-directional motion.

The kinematic model of the mobile robot is depicted in Fig.1(right). The coordinates of the mobile robot relative to a reference world coordinate system $\Sigma_{w}$ are denoted as

$$
\vec{x}=(x, y, \theta)^{T},
$$

where $x$ and $y$ are the coordinates of the intersection point $C$ of the imaginary axes connecting the wheel centers of each pair for the parallel wheels, and $\theta$ is the orientation of the mobile robot. Let, $R$ denotes the wheel radius and $L$ denotes the distance between the point $C$ and wheel center. The robot's velocity relative to $\Sigma_{r}$ is

$$
\overrightarrow{\dot{x}}_{r}=\vec{J}_{r} \overrightarrow{\dot{q}}
$$

where,

$$
\overrightarrow{\dot{x}}_{r}=\left(\dot{x}_{r}, \dot{y}_{r}, \dot{\theta}_{r}\right)^{T}, \quad \overrightarrow{\dot{q}}_{r}=\left(\dot{q}_{1}, \dot{q}_{2}, \dot{q}_{3}\right)^{T}
$$

represents velocities of the actuators, and $\vec{J}_{r}$ denotes the 
Jacobi matrix with respect to $\Sigma_{r}$ :

$$
\vec{J}_{r}=\left(\begin{array}{ccc}
k_{1} R & 0 & 0 \\
0 & k_{1} R & 0 \\
0 & 0 & k_{2} \frac{R}{L}
\end{array}\right)
$$

where, $k_{1}$ and $k_{2}$ are the gear coefficients. Because $\vec{J}_{r}$ is diagonal, the translation along each direction and rotation with respect to $\Sigma_{r}$ is decoupled and can be driven by the corresponding actuators. The mobile robot's velocity relative to $\Sigma_{w}$ is

$$
\overrightarrow{\dot{x}}=\vec{J}(\theta) \overrightarrow{\dot{q}},
$$

where $\vec{J}(\theta)$ is the Jacobi matrix with respect to $\Sigma_{w}$ :

$$
\vec{J}(\theta)=\left(\begin{array}{ccc}
k_{1} R \cos \theta & -k_{1} R \sin \theta & 0 \\
k_{1} R \sin \theta & k_{1} R \cos \theta & 0 \\
0 & 0 & k_{2} \frac{R}{L}
\end{array}\right)
$$

The equation (5) allows the evaluation of the translational and rotational velocities of the robot and the estimation of its location with respect to $\Sigma_{r}$. Equation (5) is valid for a mobile robot moving on flat ground with a pure rolling contact without slippage between its wheels and the ground.

\section{Navigation Strategy}

Our approach to integrated path planning and collision avoidance is in the five-dimensional $\left(x, y, \theta, v_{t}, v_{r}\right)$ state space in which a trade-off between computation time and collision risk is considered. $v_{t}$ and $v_{r}$ are the tangentional and rotational velocities, respectively. Unfortunately, planning in the whole state space is too time-consuming and cannot cope with the real-time constraints imposed by a mobile robot moving at high-speed in dynamic environments. Our system therefore employs different strategies to deal with the huge size of the space that should be explored as well as the dynamics of the environment. To represent the environment, our system uses occupancy grid maps [25]. This representation separates the environment into a grid of equally spaced cells and stores the probability $P(x, y)$ occupied by an object in each cell $(x, y)$. To integrate sensory input into this map we apply a Dempster-Shafer theory [26] to maintain the map from ultrasonic sensors' reading. In our approach, the path from the current location of the robot to a target position in the $(x, y)$ space is obtained through from the gradient method [8] in order to find the shortest path.

\subsection{Environment Map Building}

As stated above, our method represents the environment model of the mobile robot using an occupancy grid map. In unknown environment, the environment model is constructed based on sensor measurements. This section describes how our method can update and maintain the model of an environment using ultrasonic sensors that is equipped on the mobile robot. Occupancy grid maps represent environments by equally spaced grids. Each grid cell contains a value that indicates the presence or absence of an obstacle in the corresponding region of the environment. The occupancy grid maps are originally proposed in [25] and implemented successfully in various systems. Each cell $(x, y)$ in the map has an occupancy value $p_{\text {occ }}(x, y)$, which measures the mobile robot's belief whether or not its center can be moved to the center of the cell. To consider a size and shape of the mobile robot, the occupancy map models the configuration space of the mobile robot [27].

The ultrasonic sensor used in our approach is a range measurement device which obtains range measurement exclusively via time-of-flight(TOF) information. Map building using ultrasonic sensors has been addressed by many researchers [24],[28],[29]. The ultrasonic sensor's popularity may be attributed to its diverse availability. However, a number of its characteristics have made it less attractive for map building, whose weakest points are on the width of the beam, the specular properties of the environment and low bandwidth. Since, after transmitted, an echo is some time later due to the width of the beam, it is difficult to ascertain what part of the beam has caused the echo to return.
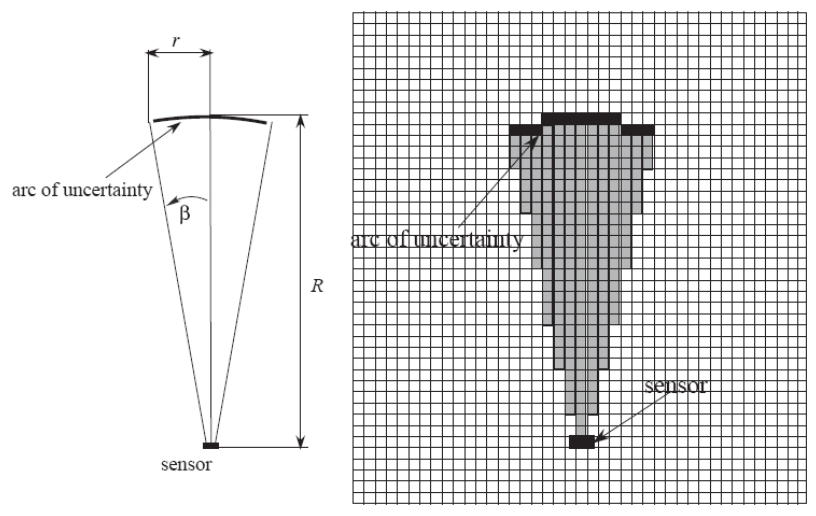

Fig. 3 Ultrasonic sensor with beam angle

Clearly, if thresh holding occurs, the only available evidence that can be ascertained from a single chirp is the range of an obstacle and the heading angle of the sensor. The only valid assumption that can be made is that the echo is generated somewhere on an arc at range, within the sensor beam $\pm \beta$ in Fig. 3.

To filter out the inherent uncertainties of the ultrasonic sensor we have focused on the probabilistic method of evaluation for the sensor confidence. Under the constraint that there is only one source of an echo along the arc, the sum of 
probabilities of all cells over the sensor arc being occupied(occ) is equal to 1 :

$$
\sum_{\forall c e l l s(i, j) \in \text { arc }} P_{o c c}(i, j)=1
$$

where $i$ and $j$ are the elements of coordinates in $x$ and $y$, respectively.

Furthermore, the arc of the sensor scan provides no information about the negation. Therefore, the probability of mass distribution along the uncertainty arc is

$$
P_{o c c}(i, j)=\frac{1}{2 R \beta}, \quad \forall \operatorname{cell}(i, j) \in \operatorname{arc}
$$

where $R$ is the range and $\beta$ is the beam angle.

By projecting the sensor beam onto a rectangular grid of discrete cells in equal size, $n$ cell is affected by the sensor arc

$$
P_{o c c}(i, j)=\frac{1}{n}, \quad \forall \text { cells }(i, j) \in \operatorname{arc} .
$$

The probabilities over the sensor arc are equally assigned, and there is no evidence about the negation, therefore,

$$
P_{\text {emp }}(i, j)=0, \quad \forall \text { cells }(i, j) \in \operatorname{arc} .
$$

When $P_{o c c}(i, j)+P_{e m p}(i, j) \neq 1$, we diverge from the Bayesian theory requirement. Similarly, for the unoccupied sector bounded by $\pm \beta$ and its arc, there is no information about the probability of the cells being occupied

$$
P_{o c c}(i, j)=0, \quad \forall \text { cells }(i, j) \in \text { sector. }
$$

The probability of being empty being with a constant all over the sector, concurrently with the lack of evidence about the cells being occupied is

$$
P_{\text {emp }}(i, j)=\rho, \quad \forall \text { cells }(i, j) \in \text { sector } .
$$

Because of these properties, the ultrasonic range readings are well suited to the application of the Dempster-Shafer that has the probabilities $P_{o c c}(i, j)$ and $P_{e m p}(i, j)$ corresponding to the basic probability assignments $m_{i, j}\left(\right.$ occ) and $m_{i, j}(e m p)$ [26].

To build an occupancy map of the environment, we construct a grid representing the environment. Each cell in the grid is characterized by two states : occupied and empty. Therefore, we define the field of discernment, $\Theta$ by the set $\Theta=\{O, E\}$ where $O$ and $E$ correspond to the probabilities. The cell is either occupied or empty, respectively. The set of all subsets of $\Theta$ is the power set

$$
\Omega=2^{\Theta}=\{O, E,\{O, E\}\} .
$$

The state of each cell is described by assigning basic probability mass to each label in $\Omega$, in which

$$
\sum_{A \subset \Omega} m_{i, j}(A)=m_{i, j}(O)+m_{i, j}(E)+m_{i, j}(\{O, E\})=1 .
$$

It is sufficient to store only $m_{i, j}(O)$ and $m_{i, j}(E)$ in order to represent the state of the system. Every cell in the map is first initialized, $m_{i, j}(O)=m_{i, j}(E)=0$ and $m_{i, j}(\{O, E\})=1$, representing total ignorance about the state of each cell. As the mobile robot moves, scans of the environment are taken and fused into the map. The errors less than the resolution of the map in the position of a mobile robot are absorbed by the cell size.

The basic probability assignment for the sensor arc are

$$
\left.\begin{array}{l}
m_{i, j}(O)=\frac{1}{n} \\
m_{i, j}(E)=0
\end{array}\right\} \quad \forall \text { cells }(i, j) \in \operatorname{arc}
$$

within the sector

$$
\left.\begin{array}{l}
m_{i, j}(O)=0 \\
m_{i, j}(E)=\rho
\end{array}\right\} \quad \forall \text { cells }(i, j) \in \text { sector }
$$

and outside the sector

$$
\left.\begin{array}{l}
m_{i, j}(O)=0 \\
m_{i, j}(E)=0
\end{array}\right\} \quad \forall \text { cells }(i, j) \notin \text { arc, sector. }
$$

The basic probability mass assigned to the empty cells is constant and equal to the masses for the occupied cells on the arc $\frac{1}{n}$. However, large range readings will cause low basic probability assignments since $n$ is relatively large. Finally, each cell in the map is updated using the following Dempster's rule of combination

$$
m_{1} \oplus m_{2}(A)=\frac{\sum_{\forall B, C \in \Omega: B \cap C=A} m_{1}(B) m_{2}(C)}{1-\sum_{\forall B, C \in \Omega: B \cap C=\varphi} m_{1}(B) m_{2}(C)}
$$

where subscripts 1 and 2 represent the basic probability masses $m$ according to sensor and map, respectively.

If $A$ is the evidence of empty, $B$ and $C$ are evidences indicating empty $E$ and the ignorance $\{O, E\}$ from the perspective of sensor reading and map. Thus, the combination should be done in both way, updating empty state and occupancy state. The ignorance can be derived from basis probability masses of $E$ and $O$. Note that this formula can be used to update occupancy values incrementally. The final occupation value attributed to a cell is given by comparing the empty and occupied basic probability masses. 


$$
\operatorname{map}(i, j)=\left\{\begin{array}{c}
m_{i, j}(O) \quad \text { if } m_{i, j}(O) \geq m_{i, j}(E) \\
-m_{i, j}(E) \text { if } m_{i, j}(O)<m_{i, j}(E)
\end{array} .\right.
$$

\subsection{Goal-directed Obstacle Avoidance}

The path planning process is producing a minimum-cost path to the goal. The cost of traversing a grid cell is based on a combination of intrinsic costs defined using occupancy value and predefined path costs. The path cost can be defined using a generalized wave propagation algorithm that makes a function with a single minimum at the goal configuration. The minimum-cost path is computed using a dynamic programming algorithm. One of successive navigation techniques based on this method is a gradient method [8]. The mobile robot finds the shortest path and follows the descendent gradient of the navigation function to reach a goal. The method can take a goal point as an input, the final point of the computed path will be the best one with respect to the navigation function. The method lies in the formulation of multiple layers of costs, i.e., how the intrinsic cost can be represented and how the layers can be combined. A path is defined as an ordered set of sampling points

$$
P_{n}=\left\{p_{n}, p_{n-1}, \ldots, p_{0}\right\}
$$

where $p_{i} \in \mathfrak{R}^{2}, \forall i=n, \ldots, 1$ and $p_{i}$ must be adjacent to $p_{i-1}$. Therefore, if $i \neq j, \forall i, j$ then $p_{i} \neq p_{j}$ The $p_{0}$ must be in the set of the final configurations, while $p_{i}, \forall i=n, \ldots, 1$ must not be in the set of the final configurations.

Given a point $p_{k}$, a path that starts from $p_{k}$ and reaches one of the final configuration $p_{0}$ will be represented as $P_{k}=\left\{p_{k}, \ldots, p_{0}\right\}$. A cost function for a path $P$ is $F(P)$, an arbitrary function of the (discrete) path. This function is the sum of an intrinsic cost $I$ and an adjacency cost $A$ :

$$
F\left(P_{k}\right)=\sum_{i=0}^{k} I\left(p_{i}\right)+\sum_{i=0}^{k-1} A\left(p_{i}, p_{i+1}\right) .
$$

Both $I$ and $A$ can be arbitrary functions. $I$ depends on how close the mobile robot is to obstacles, while $A$ is proportional to Euclidean distance between two points.

The value of the navigation function $N_{k}$ in a point $p_{k}$ is the assignment of a potential field value to every element of the configuration space, such that the goal set is always "down hill" no matter where the mobile robot are in the space. $N_{k}$ is the cost values for a minimum path that starts from $p_{k}$ :

$$
N_{k}=\min _{j=1,, m} F\left(P_{k}^{j}\right)
$$

where $P_{k}^{j}$ is the $j$-th path starting from point $p_{k}$ and reaching one of the final destinations, and $m$ is the number of such paths.
Calculating the navigation function $N_{k}$ directly for every point in the configuration space, would require a very high computational time, even for a small configuration space. To compute the navigation function efficiently, the computation is proceeded by the following steps:

1. Assign the grid cell that contains the final configuration with and the other with

$$
F(x, y)=\left\{\begin{array}{c}
0, \text { if }(x, y) \text { is the final configuration } \\
\infty, \quad \text { otherwise }
\end{array}\right.
$$

2. At each iteration, operate on each point by updating its 8 neighbors

$$
F(x, y)=\min \{I(x+\zeta, y+\xi)+A(x+\zeta, y+\xi)\}
$$

where $\zeta=-1,0,1$ and $\xi=-1,0,1$.

\subsection{On-line parameter adaptation}

In this section, we describe a method for changing parameters based on sensor data. This paper deals with the model based motion planning using navigation function [8] and an autonomous robot equipped with 8 sonar sensors. We focus on narrowness of a passage and notice that sensor data indicate narrowness of a passage. Since it is not easy to deal with all sensor data, we consider which sonar data should be chosen among 8 sonar sensors. Then we define $S_{\min }$ as a sensor data from the closest sonar to obstacles and $S_{m o v}$ as a sensor data on moving direction and $S_{s y m}$ as a sensor data with a symmetric relation with $S_{\min }$ to moving direction in Fig. 5. Consider that these three variables indicate the narrowness of a passage, and the repulsive potential $I$ is composed by these variables.

$$
I=I\left(S_{m o v}, S_{\min }, S_{s y m}\right)
$$

In simplest case, $I$ is a function of only

$$
I=I\left(S_{s y m}\right) \text {. }
$$

It is not difficult to see that this function indicates narrowness of passage. For example, $I$ is proportional to $S_{s y m}$. In this case, for large $S_{s y m}$ a robot moves far from obstacles because $I$ is also large. On the other hand, for small $S_{s y m}$ a robot moves near obstacles and can pass through a narrow passage because $I$ is also small. Thus, if a robot changes parameters while its moving, then a robot can achieve both objectives for obstacle avoidance and a narrow passage problem. As well, this concept is applied to change velocity. For a narrow passage a robot moves slowly, and for a wide passage a robot moves fast. Hence, a robot can avoid collision and reach a goal in a satisfactory way. Even for U-shaped environment, at first a 
robot moves slowly since there is an obstacle on moving direction. After a robot knows there is no direct straight path on moving direction then a robot backs off and moves fast since there is no obstacle on moving direction. In following sections, U-shaped deep narrow obstacle and narrow passage situations are shown using computer simulations in order to prove the effectiveness of the proposed scheme.

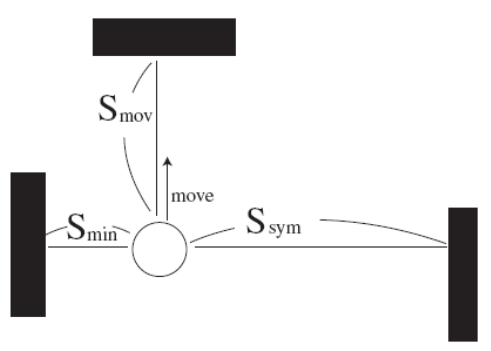

Fig. 4 Relation of sonar

\section{Simulations}

\subsection{U-shaped Deep Narrow obstacle}

The simulations of a U-shaped obstacle problem are shown in Fig. 5 and 6. The current location of the mobile robot and the potential generated for navigation are depicted in which the width between two walls are $1.2 \mathrm{~m}$ in Fig. 5 and $1.0 \mathrm{~m}$ in Fig. 6 and the depth of passage is $1.8 \mathrm{~m}$ in both situations. The goal is the brightest location in the gray window inside the right black window according to the potential. First, the robot directly goes to the goal. However when the robot detects the front wall, the robot recognizes no direct path to the goal. Then the robot goes back the path the robot has come. After that, the robot goes around a U-shaped obstacle and then finally reaches the goal. In the simulations, the path that the robot should move is generated and changed at every time step. The simulator used in the simulations is the Player/Stage [30], [31] running on 2.4GHz Pentium PC under Linux OS.

\subsection{Narrow Passage between two obstacles}

The simulations of the narrow passage situation are shown in Fig. 7 and Fig. 8. A narrow passage situation has a $1.0 \mathrm{~m}$ and $0.8 \mathrm{~m}$ gap between obstacles in Fig. 7 and Fig. 8, respectively. Although there is no high computational cost for the drastic environment changes, the accurate detection of the narrow gap based on an ultrasonic sensor array is required. Each 45 degree beam width and its rough reflection could lead to fault recognition on the narrow passage with relatively small width. In the simulations, no perception noise and action noise are assumed, so the mobile robot can perform the navigation well for two $0.6 \mathrm{~m} * 0.6 \mathrm{~m}$ obstacles. In both cases, the proposed method shows that the robot passes through the narrow passages and reaches the goal area. Even the small gap as $0.8 \mathrm{~m}$, the robot goes toward the two closely spaced obstacles without taking a detour.

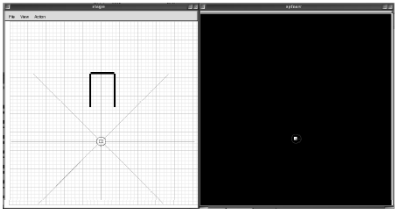

(a)

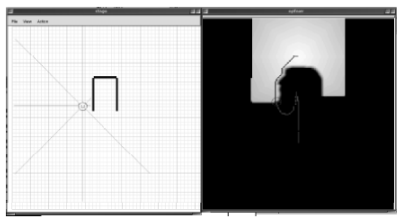

(c)

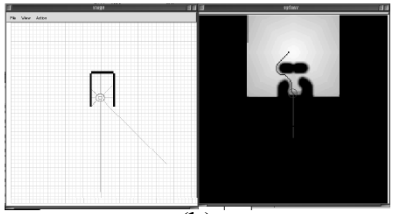

(b)

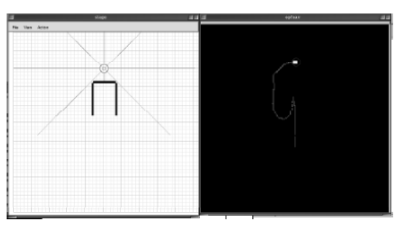

(d)
Fig. 5 A U-Shaped environment with the depth of $1.8 \mathrm{~m}$ and width of $1.2 \mathrm{~m}$.

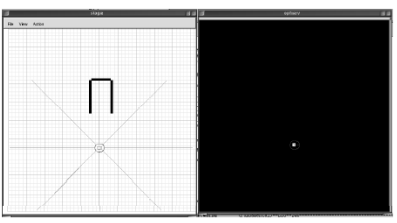

(a)

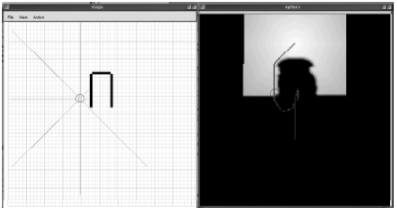

(c)

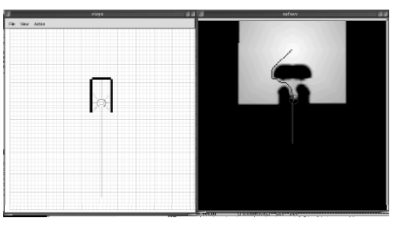

(b)

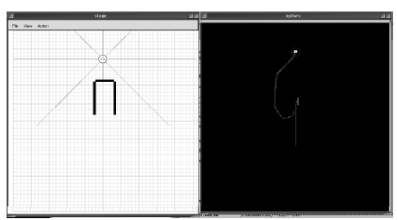

(d)
Fig. 6 A U-Shaped environment with the depth of $1.8 \mathrm{~m}$ and width of $1.0 \mathrm{~m}$.

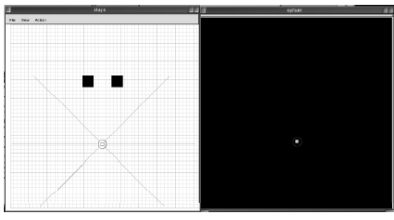

(a)

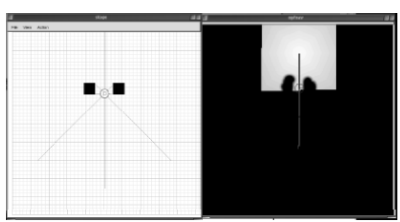

(c)

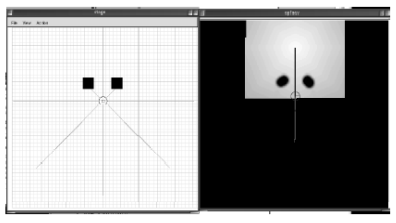

(b)

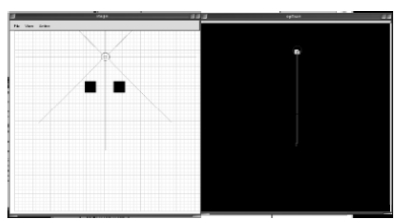

(d)
Fig. 7 A narrow passage situation with a $1.0 \mathrm{~m}$ gap between obstacles. The dimension of an obstacle is $0.6 \mathrm{~m} \times 0.6 \mathrm{~m}$. 


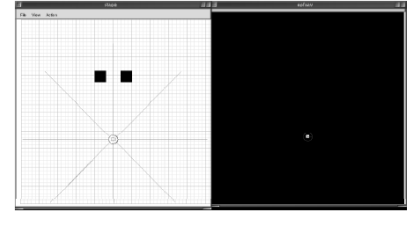

(a)

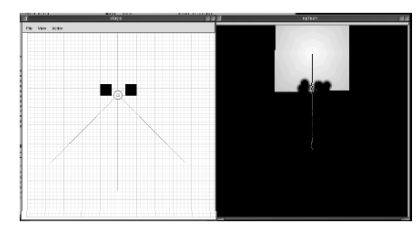

(c)

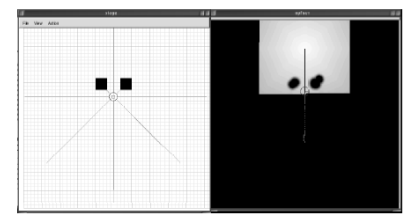

(b)

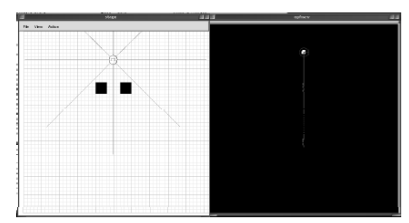

(d)
Fig. 8 A narrow passage situation with a $0.8 \mathrm{~m}$ gap between obstacles. The dimension of an obstacle is $0.6 \mathrm{~m} \times 0.6 \mathrm{~m}$.

\section{Conclusions}

This paper presents a navigation framework based on a descendent gradient method for an omni-directional mobile robot in unknown environment. The main objective is to overcome some of the typical problems that may pose to the conventional robot navigation through the dynamic environment model building and efficient navigation strategy. The proposed navigation scheme eliminates the non-feasible area for the two cases by the help of the descendent gradient of the navigation function and the characteristics of an omnidirectional mobile robot. As well, different from previous studies on navigation strategy, this study solves the U-shaped deep narrow obstacle problem caused by goal-directed navigation approaches and the narrow passage problem between two obstacles effectively, and investigate the navigation ability in a trapped environment depending on sensor range. As for the problems, simulation results are presented in the realistic environment. Compared to the conventional algorithms for such a sensor based navigation, rather simple algorithm is used to solve the proposed problems. This approach is realistic and more natural because the robot moves with keeping off the obstacles but still it has a good reachability to the goal while highly depending on sensor range. Further research is on the experimental results in narrow passages or in the presence of obstacles.

\section{References}

[1] P. Stepan, L. Kral , M. Kulich, and L. Preucil, "Open control architecture for mobile robot," Proc. of 14th World Congress of IFAC, pp. 163-168, 1999.

[2] B. Wielinga and G. Schreiber, "Configuration-design problem solving, " IEEE Expert , pp. 49-56, 1997.

[3] J. C. Latombe, "Robot Motion Planning," Boston, MA:Kluer, 1991.
[4] W. Kevin and A. Bowyer, "A survey of configuration-space mapping techniques for a single robot in a static environment," Int. Journal of Robotics Research, vol. 8, pp. 762-779, 2000.

[5] E. Rimon and D. E. Koditschek, "Exact robot navigation using artificial potential functions, "IEEE Trans. on Robotics and Automation, vol. 5, pp. 501-518, 1992.

[6] Y. Koren and J. Borenstein, "Potential field methods and their inherent limitations for mobile robot navigation," Proc. of the IEEE int. Conf. on Robotics \& Automation, pp. 1398-1404, 1991.

[7] H. Asama, M. Sato, L. Bogoni, H. Kaetsu, A. Matsumoto, and I. Endo, "Development of an Omni-Directional Mobile Robot with 3 DOF Decoupling Drive Mechanism," in Proc. of the IEEE Int. Conf. on Robotics and Automation, Nagoya, Japan, pp. 1925-1930, 1995.

[8] K. Konolige, "A gradient method for realtime robot control," Proc. of the IEEE/RSJ Int. Conf. on Intelligent Robots and Systems (IROS), 2000.

[9] J.-C. Latombe, "Robot Motion Planning.," Kluwer Academic Publishers, 1991.

[10]O. Khatib, "Real-time Obstacle Avoidance for Robot Manipulator and Mobile Robots, " The International Journal of Robotics Research, vol. 1, pp. 90-98, 1986.

[11]J. Borenstein and Y. Koren, "The Vector field Histogram-Fast Obstacle Avoidance for Mobile Robots," IEEE Trans. on Robotics and Automation, vol. 3, pp. 278-288, 1991.

[12]D. Fox, W. Burgard, and S. Thrun, "A hybrid collision avoidance method for mobile robots," in Proc. of the IEEE Int. Conf. on Robotics \& Automation(ICRA), 1998.

[13]D. Fox, W. Burgard, and S. Thrun, "The Dynamic Window Approach to Collision Avoidance," IEEE Robotics \& Automation Magazine, vol. 1, 1997.

[14] O. Brock and O. Khatib, "High-speed navigation using the global dynamic window approach," in Proc. of the IEEE Int. Conf. on Robotics \& Automation(ICRA), 1999.

[15]M. Khatib and R. Chatila. "An Extended Potential Field Approach for Mobile Robot Sensor-based Motions," in Proc. of Int. Conf. on Itelligent Autonomous Systems(IAS'4), 1995.

[16] N. Y. Ko and R. Simmons, "The Lane-curvature Method for Local Obstacle Avoidance," in Proc. of the IEEE/RSJ Int. Conf. on Intelligent Robots and Systems(IROS), 1998.

[17]C. Trevai, R. Takemoto, Y. Fukazawa, J. Ota and T. Arai. "Local Obstacle Avoidance with Reliable Goal Acquisition for Mobile Robots," Distributed Autonomous Robotics Systems(DARS04), 2004.

[18]C. Schlegel. "Fast Local Obstacle Avoidance under Kinematic and Dynamic Constraints for a Mobile Robot," in Proc. of the IEEE/RSJ Int. Conf. on Intelligent Robots and Systems(IROS), 1998.

[19] J. Buhmann, W. Burgard, A.B. Cremers, D. Fox, T Hofmann, F. Schneider, J. Strikos and S. Thrun. "The Mobile Robot 
RHINO," AI Magazine,vol. 1, 1995.

[20]L. Kavraki, P. Svestka, J.-C. Latombe and M. Overmars. "Probabilistic Roadmaps for Path Planning in High Dimensional Configuration Spaces," IEEE Transactions on Robotics and Automation, vol. 4, pp. 566-580, 1996.

[21]I. Ulrich and J. Borenstein. "VFH+: Reliable Obstacle Avoidance for Fast Mobile Robots, "In Proc. of the 1998 IEEE Int. Conf. on Robotics and Automation, pp. 1572-1577, 1998.

[22] W. Burgard, A.B. Cremers, D. Fox, D. Hähnel, G. Lakemeyer, D. Schulz, W. Steiner, and S. Thrun. "Experiences with an Interactive Museum Tour-guide Robot," Artificial Intelligence, vol. 1-2, 2000.

[23] S. Thrun. A. Bücken, W. Burgard, D. Fox, T. Fröhlinghaus, D. Henning, T. Hofmann, M. Krell, and T. Schimdt. "Map Learning and High-Speed Navigation in RHINO. In D. Kortenkamp, R.P. Bonasso and R. Murphy, editors, AI-Based Mobile Robots: Case Studies of Successful Robot Systems," MIT Press, Cambridge, MA, 1998.

[24] A. Elfes, "A Sonar-Based Mapping and Navigation System. in Carnegie-Mellon University," The Robotics Institute, Technical Report, pp. 25-30, 1985.

[25]H.P. Moravec and A.Elfes, "High Resolution Maps from Wide Angle Sonar," In Proc. IEEE Int. Conf. Roboti cs and Automation, pp.116-121, 1985.

[26]D. Pagac, E. M. Nebot and H. D.-Whyte, "An Evidenti al Approach to Map-Building for Autonomous Vehicle s," IEEE Transactions on Robotics and Automation, vol. 4, 1998.
[27] J.-C.Latombe, "Robot Motion Planning," Kluwer Academic Publishers, Boston, MA, 1991.

[28]J. J. Leonard, "Directed sonar sensing for mobile robot navigation," Ph.D. dissertation, University of Oxford, Oxford, U.K., 1991.

[29]B. Barshan and R. Kuc, "Differentiation sonar reflecti ons from corners and planes by employing and intelligent sensor," IEEE Trans. Pattern Analysis. Machine Intelligence, pp. 560$569,1990$.

[30] B. P. Gerkey, R. T. Vaughan, K. Stoy, A. Howard, G. S. Sukhatme, and M. J. Mataric, "Most Valuable Player: A Robot Device Server for Distributed Control," in Proc. of the IEEE/RSJ Int. Conf. on Intelligent Robots and Systems, pp. 1226-1231, Wailea, Hawaii, 2001.

[31] B. Gerkey, R. T. Vaughan, and A. Howard. "The Player/Stage Project: Tools for Multi-Robot and Distributed Sensor Systems," in Proc. of 11th Int. Conf. on Advanced Robotics, pp. 317-323, Coimbra, Portugal, June, 2003.

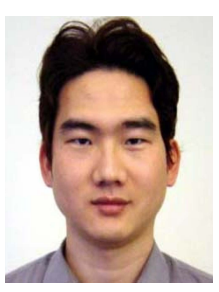

Dong Hun Kim received his B.S., M.S., and $\mathrm{Ph} . \mathrm{D}$. degrees from the Department of Electrical Engineering, Hanyang University, Korea, in 1995, 1997, and 2001, respectively. From 2001 to 2003, he was a Research Associate under several grants in the Department of Electrical and Computer Engineering, Duke University, NC, USA. In 2003, he joined Boston University, MA, USA, as a Visiting Assistant Professor under several grants in the Department of Aerospace and Mechanical Engineering. In 2004, he was engaged in postdoctoral research at the School of Information Science and Technology, the University of Tokyo, Japan. Since 2005, he has been an Associate Professor with the Department of Electrical Engineering, Kyungnam University, Korea. His research interests include swarm intelligence, swarm robotics, self-organization of swarm systems, mobile robot path planning, decentralized control of autonomous vehicles, intelligent control, and adaptive nonlinear control. 